\title{
DESALINIZADOR SOLAR DE MÚLTIPLES EFECTOS A ALTA TEMPERATURA: DISEÑO, MODELACIÓN Y SIMULACIÓN
}

\author{
Eduardo Gálvez Soto ${ }^{1}$ Pedro Roth Urban ${ }^{2} \quad$ Klemens Schwarzer $^{3}$ \\ Recibido el 09 de diciembre de 2002, aceptado el 22 de septiembre del 2003
}

\begin{abstract}
RESUMEN
La siguiente investigación consisten en diseñar, modelar y simular un desalinizador solar de múltiples efectos que trabaje a alta temperatura, el cual esté orientado a satisfacer las necesidades de agua de comunidades de pescadores artesanales aisladas en el norte de Chile. En este sentido se mezclan aspectos científicos, tecnológicos y sociales. Esto hace que la modelación considere aspectos como: un equipo compacto, modular, de bajo costo, mantención sencilla, vida útil significativa y de alta eficiencia.

El equipo desalinizador funciona con una bandeja de doble fondo y cinco bandejas principales (cinco efectos), que actúan como evaporador y condensador a la vez. La energía será suministrada a través de un colector solar conectado a un intercambiador de calor ubicado en la bandeja de doble fondo.

Se ha desarrollado un programa computacional que a través del modelo matemático, muestra el comportamiento de funcionamiento del equipo desalinizador. Este programa ha sido elaborado utilizando Matlab-Simulink® con el apoyo del software biblioteca Carnot ${ }^{\circledR}$ que ha desarrollado el Solar-Institut Jülich de la Fachhochschule de Aachen en Alemania.

Los resultados de la simulación son gráficas o bases de datos, de los diferentes comportamientos de las temperaturas, producción de condensado y/o energías involucradas.

La simulación nos lleva concluir que la producción de agua desalinizada de este equipo debe superar los $20 * 10^{-3}$ $\mathrm{m}^{3} /$ día.
\end{abstract}

Palabras claves: Destilador, colector solar, múltiples efectos

\begin{abstract}
The purpose of this work is to design, model and simulate a solar multiple effect distiller working at high temperature. The design of the equipment must satisfy the needs of water of isolated fishermen communities in the north of Chile. The basic characteristics of the system should be: low cost, simple maintenance, compact, modular, with a long useful life and high efficiency.

The model of the proposed distiller is a relatively new development in the area of the solar distillation with heat recovery. The distiller has double bottom and five main trays (five effects) that work like evaporator and condenser at the same time. The condensation heat of each stage is used in the following step. The amount of used energy can be through a solar collector connected to a heat exchanger in the tray of double bottom or using another energy source that may be adapted to the system.

A computer program has been developed, showing the behavior of operation of the equipment. This program has been realised using Matlab-Simulink $®$ with the support of the software library Carnot $囚$ that was developed by the SolarInstitut Jülich of the Fachhochschule of Aachen in Germany.

The simulation program is quite flexible, allowing modifications of the different parameters of the thermal analysis and the different equations of the model.

The results of the simulation, the behavior at different temperatures, water production and involved energy are presented in graphic and database forms.
\end{abstract}

Keywords: Distiller, solar collector, multiple effects

\footnotetext{
1 Universidad de Tarapacá, Depto. de Mecánica, Arica - Chile, Casilla 6- D, Teléfono: 56-58-205265, Fax: 56-58-205281, egalvez@uta.cl

2 Universidad Técnica Federico Sta. María, Depto. de Mecánica, Avda. España 1680, Valparaíso - Chile, Casilla 110-V, Teléfono:56-32-654178, Fax 56-32-797472.

3 Solar-Institut Jülich, Heinrich-Mußmann-Str. 5, 52428 Jülich - Germany, Teléfono: 49-02461- 99-3520, email: schwarzer@sij.fh-aachen.de
} 


\section{FUNCIONAMIENTO DEL SISTEMA DESALINIZADOR}

El Desalinizador de Múltiples Efectos propuesto es un desarrollo relativamente nuevo en el área de la destilación solar con recuperación de calor especialmente en Chile. Éste trabaja con varias bandejas ubicadas una sobre otra y utilizan el calor de condensación de cada etapa para el paso siguiente. El calor o energía de entrada es llevado a través de un colector solar al equipo de desalinización. Es Posible el uso de otras fuentes de energía.

El colector solar es un equipo que trabaja con un fluido térmico (mezcla de agua destilada-etilenglicol) capaz de conseguir temperaturas superiores a $\operatorname{los} 100{ }^{\circ} \mathrm{C}$,. Este fluido se hace circular por el interior de la bandeja de doble fondo ubicada en la parte inferior del equipo desalinizador. Se realiza pues un intercambio de calor entre el fluido térmico y el agua de mar. La separación entre los fluidos es una placa de acero inoxidable de 1 mm de espesor.

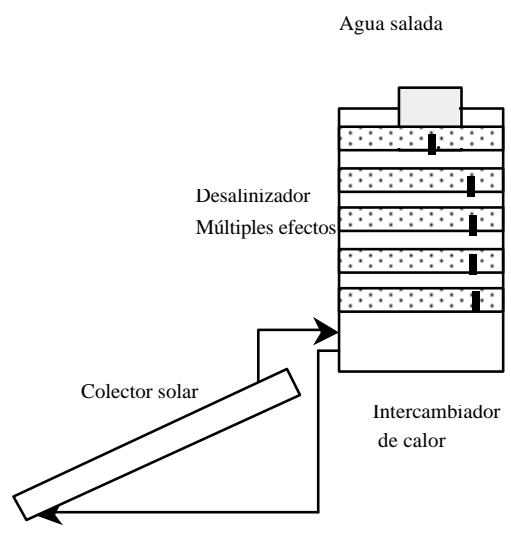

Fig. 1.- Equipo desalinizador

A través de la entrada de calor, el agua salada recibe en la etapa más baja aproximadamente 90 - $95{ }^{\circ} \mathrm{C}$, se calienta y evapora (convección libre del aire húmedo). El vapor en el asenso del aire húmedo se condensa en la parte inferior de la próxima etapa del desalinizador. El condensado se desliza, ayudado por la fuerza de gravedad, a través de la inclinación de la pared del condensador, se recoge y se lleva a través de canaletas. Estas canaletas, debido también a su inclinación, permiten la salida del agua al exterior del destilador a través de un conducto conectado a un depósito de condensado.

El agua salada es ingresada desde arriba hacia abajo en forma descendente, contraria al flujo de calor proporcionado, mediante un estanque en la parte superior. El estanque tiene un sistema de control de nivel conectado a la bandeja de doble fondo. Aquí se controla la entrada de agua de mar, es decir, si el nivel de agua de la bandeja de doble fondo baja un cierto rango establecido, entonces se acciona el sistema de control del estanque permitiendo el ingreso del agua de mar hacia la bandeja superior. De la misma forma, si el nivel de agua sobrepasa este rango, entonces se cierra el paso del agua.

A través de la condensación se libera la entalpía de vaporización [1] que se entrega a la etapa que está encima y calienta el agua salada contenida en esa etapa. Eso lleva de nuevo a la evaporación y condensación de la etapa que está mas arriba. Esto se repite hasta llegar a la última etapa.

\section{Componentes del sistema}

El nuevo desalinizador de múltiples efectos consta de una estructura de madera, aislada térmicamente del medio exterior. Está revestida, en su interior, con una cubierta de acero inoxidable AISI 316L de espesor de 1 $\mathrm{mm}$. En su interior se encuentran las bandejas principales que son también de acero inoxidable 316L de espesor de $1 \mathrm{~mm}$. En ellas se encuentra la canaleta principal con una pequeña inclinación para colectar el condensado desde la superficie inclinada de la bandeja superior. En cada una de las bandejas existe una entrada del agua de alimentación así como una salida para el condensado a través de un tubo conectado a la canaleta principal. La capacidad de cada bandeja principal es de $0,035 \mathrm{~m}^{3}$ y la de doble fondo de $0,030 \mathrm{~m}^{3}$. Las dimensiones generales del destilador de múltiples efectos son de $882 \times 882 \times 1400\left(\mathrm{~mm}^{3}\right)$.

En el fondo del sistema se encuentra la bandeja de doble fondo, recipiente que contiene el agua de mar y fluido térmico proveniente del colector solar, denominado Wärmetausche (WT), caja térmica. La bandeja de doble fondo está diseñada para incorporar un sistema de colector solar, sin embargo es posible adaptar otro tipo de fuente de calor. El esquema propuesto muestra la forma del nuevo destilador de múltiples etapas.

\section{Formas de Energía}

El comportamiento global del destilador de múltiples efectos puede ser descrito por medio de las ecuaciones de conservación de energía y por las relaciones de los diferentes mecanismos de transferencia de calor [11], como son la Conducción, la Convección y la Radiación, además de las relaciones de Transferencia de masa.

Los flujos de energía involucrados son variados y se pueden apreciar en la Fig. 1. Existen flujos energéticos 
que son de mayor importancia relativa respecto a otros. Como por ejemplo el flujo de calor por evaporación es uno de los más relevantes en este estudio y el flujo de calor debido a las fugas de vapor hacia las etapas siguientes son prácticamente despreciable.

En cada una de las bandejas se pueden apreciar tres flujos de calor que son los debido a Evaporación, Convección y Radiación. Estos flujos de calor son los de entrada a la bandeja superior en cada etapa. En la bandeja de doble fondo el flujo de calor es el proveniente del colector solar. Se han considerado también los flujos de calor sensible del fluido de entrada, agua de mar, así como el de salida, agua desalinizada. En el lado derecho de la Fig. 1 se pueden apreciar los flujos de calor correspondiente a las fugas de calor por conducción a través de las paredes del equipo. Otra consideración en este análisis es el flujo de energía, a través de flujo de vapor, que puede pasar desde una bandeja a la otra por problemas de sello en cada etapa.

Basados en este análisis se puede entonces realizar un balance de energía y establecer la ecuaciones correspondientes para la modelación del sistema desalinizador.

El subíndice DF corresponde a la bandeja de doble fondo y los subíndices 1 al 5 corresponden a las bandejas 1 al 5 .

\section{Balance de Energía}

La ecuación de conservación de energía, que relaciona la variación de energía interna con los flujos de calor que entran y salen, aplicada al agua [2] y [3], en la bandeja de doble fondo y en la bandeja 1, para el acero inoxidable y el agua contenida en ella, se muestran a continuación:

\section{Definición de flujos de energía en el desalinizador}

Q $\quad$ : Calor por evaporación entre el agua y la placa inferior exterior

$\mathrm{Q}_{\mathrm{c}} \quad$ : Calor por convección entre el agua y la placa inferior exterior

Q. $\quad$ : Calor por radiación térmica entre el agua y la placa inferior exterior

$\mathrm{Q}_{\text {colector }}$ : Calor debido al colector solar

Qss : Calor sensible del destilado

$\mathrm{Q}_{\mathrm{se}} \quad$ : Calor sensible del agua de alimentación
$\mathrm{Q}_{\mathrm{fc}}$ : Calor por conducción por los lados del destilador

$\mathrm{Q}_{\mathrm{f}} \quad$ : Calor transferido a la siguiente etapa por fuga de vapor

$\mathrm{Q}_{\text {techo }}$ : Calor por Conducción por el techo

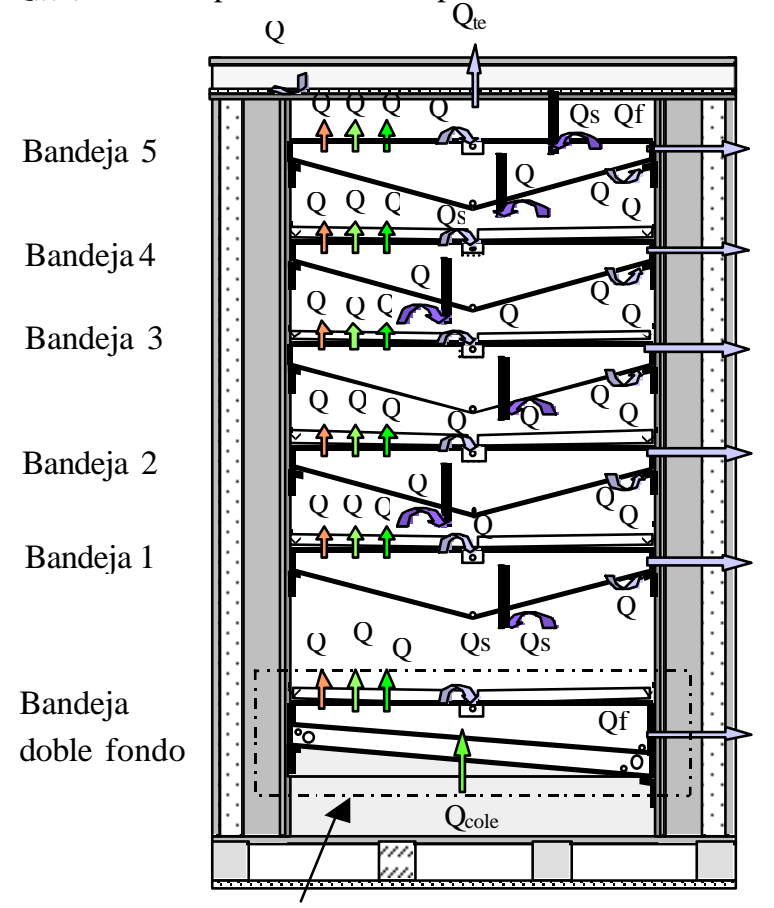

Volumen de Control

Fig. 2.- Balance de energía del desalinizador

$$
\begin{aligned}
& \mathrm{m}_{\mathrm{w}, \mathrm{DF}} * \mathrm{c}_{\mathrm{pw}} \frac{\mathrm{dT}_{\mathrm{w}, \mathrm{DF}}}{\mathrm{dt}}= \mathrm{Q}_{\text {colector }}+\mathrm{Qse}_{\mathrm{DF}}-\mathrm{Qe}_{\mathrm{DF}} \\
&-\mathrm{Qr}_{\mathrm{DF}}-\mathrm{Qc}_{\mathrm{DF}}-\mathrm{Qfc}_{\mathrm{DF}} \\
&-\mathrm{Qf}_{\mathrm{DF}}-\mathrm{Qss}_{\mathrm{DF}} \\
& \mathrm{m}_{\mathrm{t}} * \mathrm{c}_{\mathrm{pt}} \frac{\mathrm{dT}}{\mathrm{dt}}=\mathrm{Qe}_{\mathrm{DF}}+\mathrm{Qr}_{\mathrm{DF}} \\
&+\mathrm{Qc}_{\mathrm{DF}}+\mathrm{Qse}_{\mathrm{t}}-\mathrm{Qss}_{\mathrm{t}}
\end{aligned}
$$

donde,

$\mathrm{C}_{\mathrm{pw}} \quad$ : Calor específico del agua

$\mathrm{C}_{\mathrm{pt}}$ : Calor específico del acero inoxidable

$\mathrm{m}_{\mathrm{W}} \quad$ : Masa de agua en la bandeja de doble fondo

$\mathrm{m}_{\mathrm{t}} \quad$ : Masa del acero inoxidable en intercambio térmico y agua contenida en la bandeja

Tw : Temperatura del agua en la bandeja

$\mathrm{Tt} \quad$ : Temperatura de la bandeja 
El subíndice $\mathrm{t}$ indica lo relativo a la bandeja en la superficie de acero inoxidable.

Estas mismas ecuaciones aplicadas a cada una de las etapas, con la precaución de considerar solamente los flujos de calor involucrados en cada una de ellas, nos entrega el modelo matemático para el nuevo desalinizador de múltiples etapas. Este análisis nos permite determinar la temperatura del agua en la bandeja de doble fondo, la cual depende de la temperatura de la bandeja 1. Al aplicar el análisis a la segunda etapa, es decir, entre la bandeja 1 y la bandeja 2 , se puede determinar la temperatura de la bandeja 1 la cual a su vez depende de la temperatura de la bandeja 3. El análisis se aplica a cada una de las etapas en forma sucesiva, existiendo siempre una dependencia de las temperaturas.

Para determinar la temperatura de la bandeja de doble fondo, el modelo determina la temperatura de la última bandeja luego la temperatura de la penúltima y así sucesivamente hasta llegar en definitiva a la temperatura deseada.

Para el análisis de la energía que fluye a través del techo se considera la energía que proporciona la bandeja 5 y la convección exterior del equipo. La bandeja 5 debe mantener una temperatura más baja que todo el sistema. Por esta razón no se debe utilizar aislamiento en el techo.

\section{ECUACIONES DE TRANSFERENCIA DECALOR}

\section{Ecuaciones para el Calor por Evaporación}

La transferencia de calor por evaporación es una transferencia de calor asociada a la transferencia de masa, es decir, a la evaporación y condensación del agua. Existen varias formas para determinar el calor por evaporación [1], [6], [7], [8], [9], [10], dependiendo de las condiciones que se presenten. Como por ejemplo la forma Newtoniana, Kumar y Tiwari (1996), O. Headley (1977). Para el análisis del modelo se han considerado todas estas formas, y la que ha presentado el mejor comportamiento es la de E. Sartori (1996), que es la que a continuación se enuncia.

\section{Coeficientes de transferencia de calor por evaporación}

El coeficiente de transferencia de calor por evaporación puede ser determinado por la expresión (4), [8]:
Donde:

$$
\text { Qe }=60.78 * 10^{-7} * \mathrm{~B} * \mathrm{C}
$$

$$
\begin{gathered}
\mathrm{B}=\left[\mathrm{T}_{\mathrm{w}}-\mathrm{T}_{\mathrm{t}}+\left(\frac{\mathrm{P}_{\mathrm{w}}-\mathrm{P}_{\mathrm{t}}}{267.7 * 10^{3}-\mathrm{P}_{\mathrm{w}}}\right) *\left(\mathrm{~T}_{\mathrm{w}}+273.15\right)\right]^{1 / 3} \\
\mathrm{C}=\left(\mathrm{P}_{\mathrm{w}}-\mathrm{P}_{\mathrm{t}}\right) * \mathrm{~L} \\
h e=\frac{Q e}{A_{w}\left(T_{w}-T_{t}\right)}
\end{gathered}
$$

Donde Qe es el calor por evaporación y se determina usando la expresión desarrollada por Kumar y Tiwari (1996)

$$
\begin{aligned}
\bigotimes_{\mathrm{e}}= & 0.016273 *\left(\mathrm{P}_{\mathrm{w}}-\mathrm{P}_{\mathrm{g}}\right) * \\
& * \frac{\mathrm{K}_{\mathrm{wa}}}{\mathrm{d}_{\mathrm{ss}}} * 0.0538 *(\mathrm{Gr} * \mathrm{Pr})^{0.384}
\end{aligned}
$$

y donde

$$
G r=\frac{\beta^{\prime} \rho^{2} d_{s s}^{3} \Delta T^{\prime}}{\mu^{2}}
$$

$$
\Delta T^{\prime}=\left(T_{w}-T_{t}\right)+\left[\frac{\left(P_{w}-P_{g}\right)\left(T_{w}+273.15\right)}{268.9 * 10^{3}-P_{w}}\right]
$$

$$
\operatorname{Pr}=\frac{\mu * c_{p}}{K_{a w}}
$$

Muchas expresiones usadas en la determinación de los coeficientes de transferencia por evaporación están limitadas en cuanto a la temperatura de trabajo como son las expresiones propuestas por Malik (1982), esto es, válidas sólo hasta aproximadamente los $90{ }^{\circ} \mathrm{C}$. Las expresiones aquí presentadas no tienen esa restricción.

\section{Coeficientes de transferencia de calor por convección}

También considerando expresiones sin limitaciones de temperatura es que se desarrollan [8] las siguientes expresiones que relacionan los coeficientes de transferencia de calor evaporativo y convectivo 


$$
\begin{gathered}
h c=2 * \frac{M_{\text {mix }} * c_{p_{\text {mix }}}}{M_{w} * L} * \frac{1}{\frac{1}{\left(P_{a}\right)_{w}}+\frac{1}{\left(P_{a}\right)_{g}}} * h e \\
M_{\text {mix }}=\frac{M_{a} P_{a}+M_{w} P_{w}}{P} \\
c_{p_{\text {mix }}}=\frac{c_{p_{a}} P_{a}+c_{p_{w}} P_{w}}{P}
\end{gathered}
$$

\section{SIMULACIÓN COMPUTACIONAL}

\section{Generalidades}

La simulación ha sido realizada utilizando el ambiente Matlab Simulink ${ }^{\circledR}$ y el software de biblioteca Carnot ${ }^{\circledR}$ desarrollado en el Solar-Institut Julich, Alemania. El modelo matemático que se ha desarrollado plantea la búsqueda de soluciones para una serie de ecuaciones diferenciales en las cuales la variable principal es la temperatura de cada una de las etapas. El programa utiliza el método de paso variable Bogacki-Shampine ode23 que es un método basado en Runge-Kutta $(2,3)$.

El programa consta de una ventana principal o de entrada en donde se distinguen claramente los bloques que representan las etapas del desalinizador y los elementos necesarios para la entrada y salida de datos. Cada uno de los bloques es una subrutina desarrollada en forma independiente, pero que es dependiente de los otros bloques.

\section{Ventana principal}

La forma del programa está compuesta por 7 subsistemas principales (ver Fig. 2), cinco representan a cada una de las etapas del desalinizador, otro lo que es el fondo y uno final que representa la tapa. Además cuenta con una serie de componentes para la entrada y salida adecuada de los resultados como son archivos de datos y gráficos. La ventana principal contiene todos los bloques o sub-sistemas mencionados anteriormente designados con las letras WT, B1, B2, B3, B4, B5 y Tapa, en que cada uno de ellos representa un modelo matemático en particular.

\section{Componentes del programa de simulación}

Todos los bloques del programa funcionan de manera similar, sin embargo se debe cautelar que se utilicen correctamente las variables de cada etapa.

WT : Bloque que representa un sub-sistema para el fondo del desalinizador (Waermetausche). Este componente recibe el calor proveniente del Colector Solar. Este bloque requiere para su funcionamiento, el ingreso de la temperatura ambiente, la energía de entrada, y la temperatura del agua en la primera etapa. Como datos de salida se obtienen la temperatura del WT, la energía generada desde el WT hacia la primera etapa y el condensado producido por el WT. Al hacer doble clic en este bloque aparece una nueva configuración o sub-rutina. La Fig. 3 muestra la subrutina que permite calcular los diferentes tipos de energía involucrados ya sea el calor por evaporación, convección, radiación, conducción, sensibles y fugas. Permite también calcular el condensado producido y la temperatura del agua.

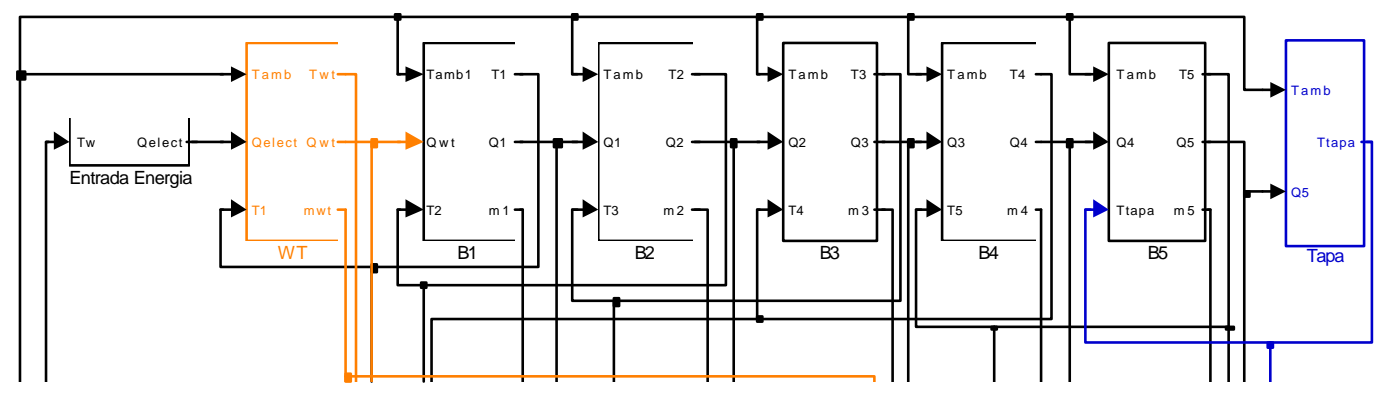

Fig. 3.- Forma de la ventana principal del programa de simulación 


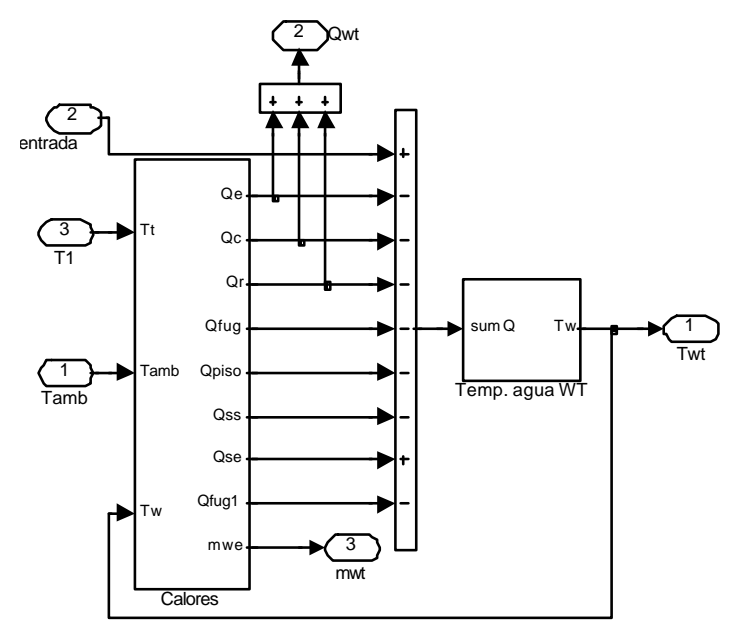

Fig.4.-Sub-rutina para la bandeja de Doble Fondo

El bloque Calores requiere para su funcionamiento la entrada de la temperatura de la primera etapa, ambiental y la del WT. La temperatura WT es determinada por el bloque "Temp. Agua WT", la temperatura de la primera etapa $\mathrm{T} 1$ es determinada en otro Bloque correspondiente a la subrutina B1 y la temperatura ambiente, Tamb, es leída desde una base de datos del bloque "Temperatura" en la ventana principal.
La salida del bloque "Calores" calcula los diferentes tipos de energía generados al interior del desalinizador. Además calcula la cantidad de condensado producido entre el WT y la primera etapa B1. para ilustrar el cálculo de los calores veremos el caso del calor por evaporación.

En la ventana de la Fig. 4 se puede apreciar el método usado para determinar el coeficiente de transferencia por evaporación, he, que es lo fundamental del cálculo de la energía, Qe, el calor debido a la evaporación. Esta subrutina puede ser modificada para incorporar otras ecuaciones para el cálculo del coeficiente de transferencia por evaporación. En esta subrutina se han utilizado bloques que son a la vez otras subrutinas para el cálculo de algunas propiedades como son: la viscosidad cinemática, la densidad, la presión de vapor, la conductividad térmica y el calor específico. Todos estos bloques extraídos desde la biblioteca Carnot.

Al despejar la temperatura en las ecuaciones del balance de energía y utilizando el método BogackiShampine ode23, se pueden encontrar los valores de $\mathbf{T}_{\mathbf{w t}} \mathrm{y}$ de $\mathbf{m}_{\mathbf{w}}$ a través del tiempo. Esto se determina en la subrutina mostrada en la Fig. 5.

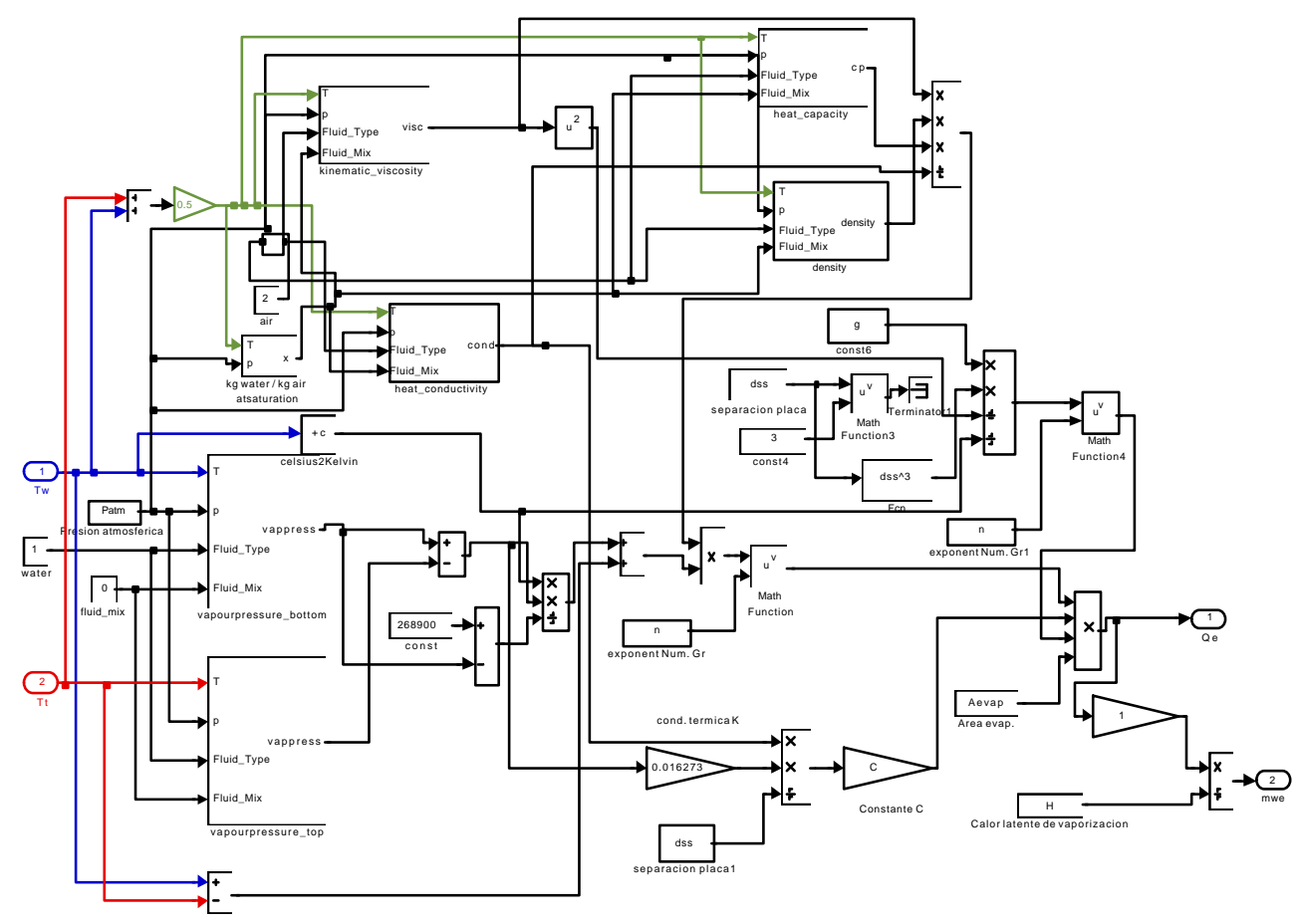

Fig. 5.- Calor por Evaporación y Cálculo del Destilado 
La salida del programa permite, entre otras posibilidades, graficar el comportamiento de la temperatura, y el condensado (Figs. 6 y 7) ya sea en forma individual como por grupo dependiendo de las condiciones que el programador establezca en el programa. Además de indicar las energías involucradas, Fig. 8.

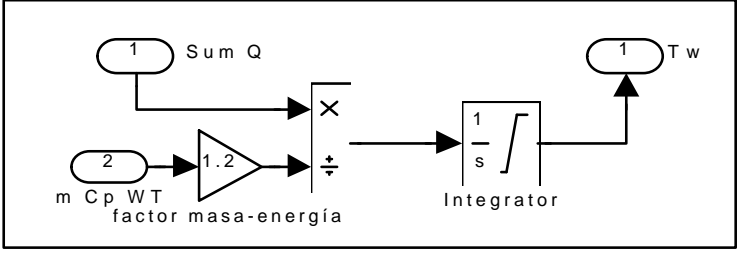

Fig. 6.- Cálculo de la temperatura del agua de la bandeja de Doble Fondo

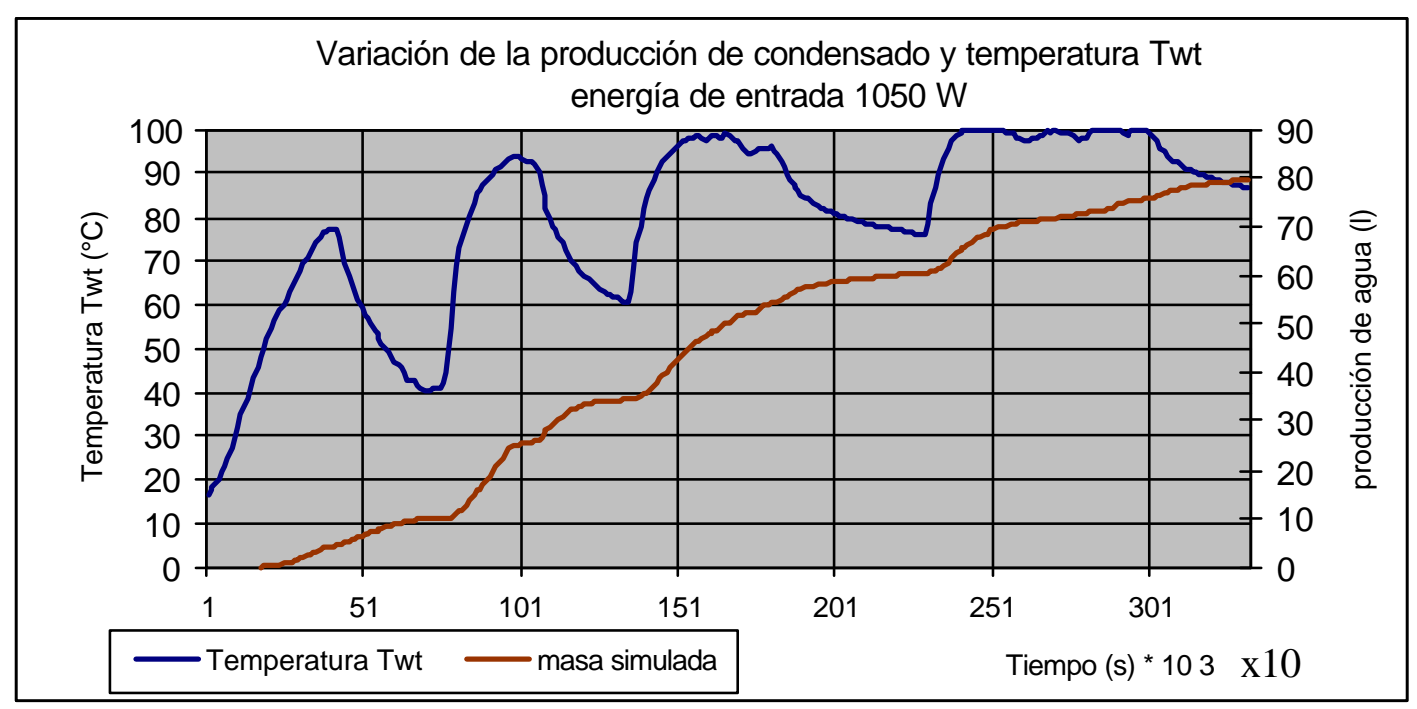

Fig. 7.- Comportamiento de la temperatura y producción de condensado

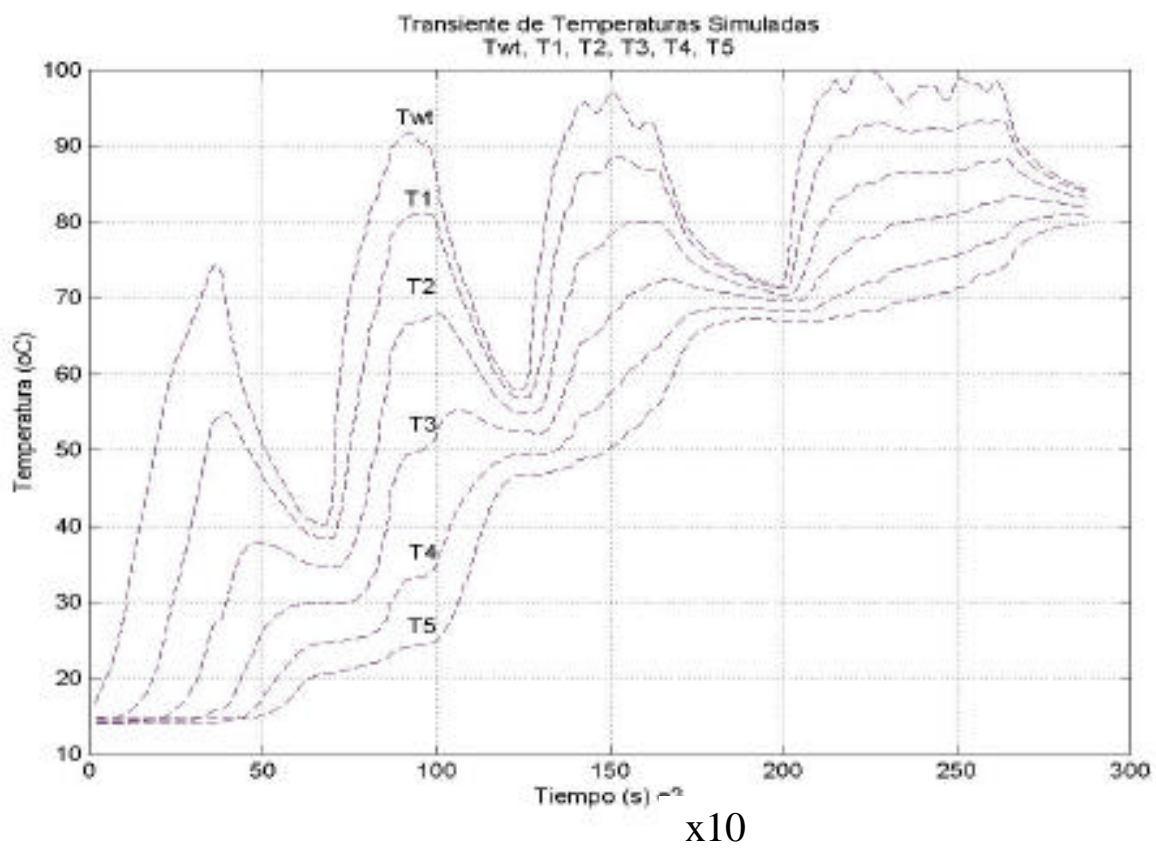

Fig. 8.- Comportamiento de la temperatura en cada efecto 


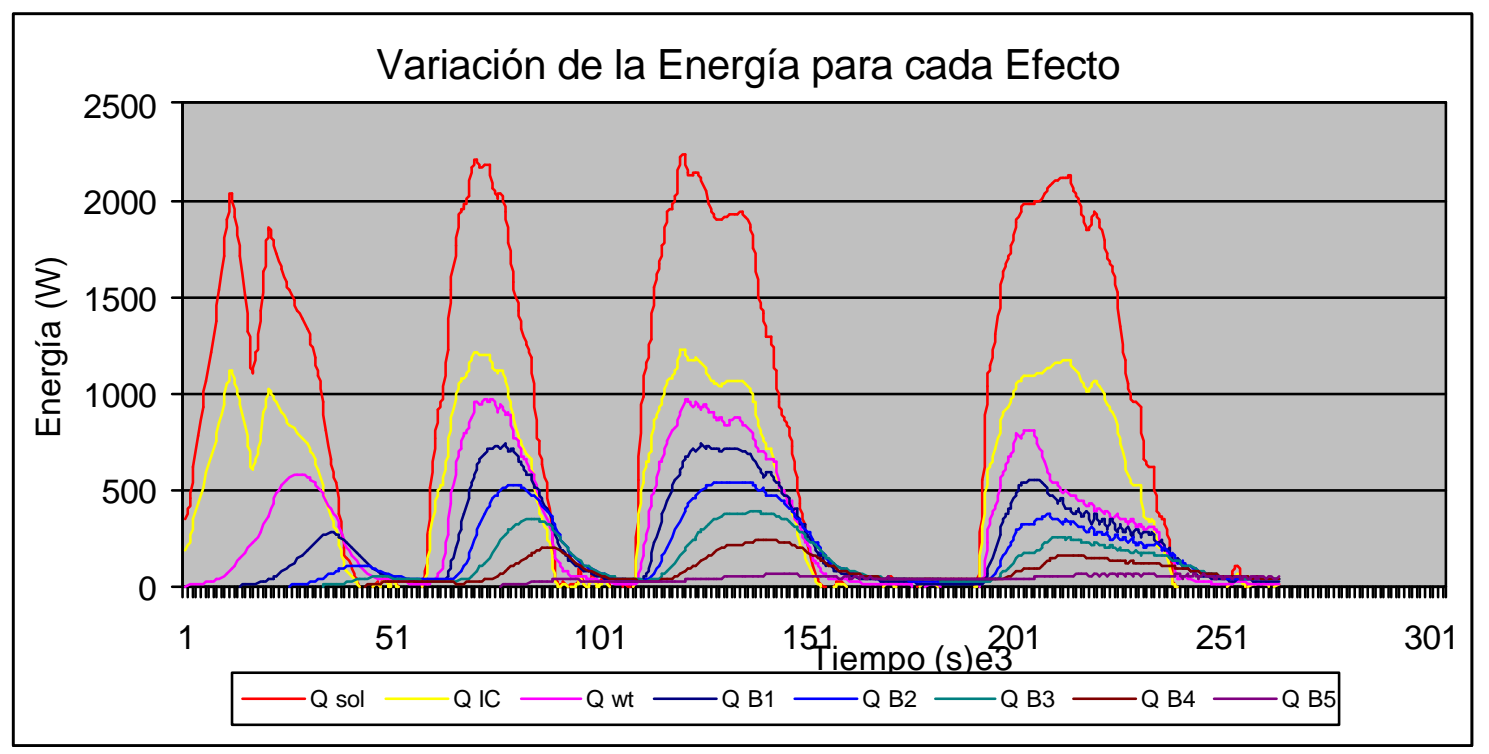

Fig. 9.- Variación de la energía en las etapas

\section{CONCLUSIONES}

Se requiere una especial atención para la formulación de un mo delo matemático que considere todos los fenómenos que ocurren y pudiesen ocurrir en un equipo desalinizador de múltiples efectos. Del análisis térmico realizado se pudieron determinar expresiones no muy sencillas que requieren de tecnología de punta para encontrar soluciones adecuadas. Es aquí donde el apoyo de investigadores del Solar-Institut Julich se hace fundamental al desarrollar un software biblioteca para la determinación de las diferentes propiedades y parámetros de los fluidos. Este software ha permitido disminuir los tiempos computacionales en la elaboración del programa de simulación.

Del transiente de temperaturas simuladas para cada una de los efectos, se establece que requiere de entre 3 a 4 días para llegar a su punto de funcionamiento óptimo.

De acuerdo a este trabajo se ha podido determinar que la participación promedio de las etapas 1, 2, 3, 4, 5, en la producción total de agua es de $30 \%, 28 \%, 22 \%, 19$ $\%$ y $1 \%$ respectivamente. Esto indica que el quinto efecto es de poca relevancia y es necesario investigar cual es el efecto sobre el equipo, si se disminuye el número de etapas.

El nivel de producción de agua del equipo desalinizador puede superar los $20 \cdot 10^{-3} \mathrm{~m}^{3} /$ día.

\section{REFERENCIAS}

[1] R. V. Dunkle; Solar water distillation: The roof type still and a multiple effect diffusion still, Commonwealth scientific and industrial research organization, C.S.I.R.O., 108, Victoria, Australia, pp. $895-902,1961$.

[2] J. A. Duffie, W. Beckmann; Solar Engineering of Thermal Processes, New York, Chichester: John Wiley \& Sons, pp. 80 - 105, 1980.

[3] M. A. S. Malik, G. N. Tiwari, A. Kumar, M. S. Sodha; Solar Distillation, Pergamon Press, pp. 5 - 89, 1982.

[4] Solar Institut Julich, Fachhochschule Aachen Abt. Julich Untersuchungen Zur Optimierung einer neurtigen Meherstufendestille zur solarthermischen Meer- und Brackwasserentsalzung fur Einzatz in Chile, Forschungsantrag im Rahmen des DFGProgrames, pp. 7-30, 1998.

[5] A. Scholz,; Optimierung einer neuartigen Mehrstufendestille zur rack- und Meerwasserentsalzung, Solar-Insttut Jülich. pp. $30-55,1999$.

[6] Sanjay Kumar y G. N. Tiwari; Estimation of convective mass transfer in solar distillation systems, Solar Energy, 57(6), pp. 459 - 464, 1996. 
[7] R. S. Adhikari, y G. D. Sootha; Simulation studies on a multi-stage stacked try still, Solar Energy, 54(5), pp 317-325, 1995.

[8] A.T. Shawaqfeh, y M. M. Farid; New development in the theory of heat and mass transfer in solar stills, Solar Energy, 55(6), pp. 527 - 535, 1995.

[9] O. Headley; Renewable energy technologies in the Caribbean, Solar Energy, 59(1-3), pp. $1-9$, 1997.

[10] E. Sartori; Solar still versus solar evaporator: a comparative study between their thermal behaviors, Solar Energy, 56(2), pp. 199 - 206, 1996.

[11] Walter Wagner,; Wärme-ubertragung, Vogel Fachbuch, p.106 - 131, 1993. 